\title{
Adaptive Nonlinear Model-Based Fault Diagnosis of Li-ion Batteries
}

\author{
A. Singh, A. Izadian, Senior Member IEEE, and S. Anwar
}

\begin{abstract}
In this paper, an adaptive fault diagnosis technique is used in Li-ion batteries. The diagnosis process consists of multiple nonlinear models representing signature faults, such as over-charge and over-discharge, causing significant model parameter variation. Impedance spectroscopy of a Li-ion ( $\mathrm{LiFePO}_{4}$ ) cell is used along with the equivalent circuit methodology to construct nonlinear battery signature fault models. Extended Kalman filters are utilized to estimate the terminal voltage of each model and to generate the residual signals. The residual signals are used in the multiple model adaptive estimation (MMAE) technique to generate probabilities that determine the signature faults. It can be seen that by using this method, signature faults can be detected accurately, thus providing an effective way of diagnosing Li-ion battery failure.
\end{abstract}

Index Terms-Li-ion Battery, Fault Diagnosis, Extended Kalman Filter, Multiple Model Adaptive Fault Diagnosis.

\section{INTRODUCTION}

$\mathrm{T}$ HE battery as an energy source has come a long way since its inception more than two centuries ago; the battery as we see it today is light in weight, packs higher energy, is safer to handle, and has a longer life [1]. Due to the inherent benefits of the technology, batteries are found in a range of consumer and industrial applications. Lithium batteries, exhibiting higher energy densities than their counterparts, have seen an unprecedented increase in production and subsequent use with the annual lithium consumption for battery production going from negligible to 6500 metric tons in 15 years, from 1993 to 2008 [2]. Lithiumion, the rechargeable counterparts of lithium batteries, are widely used in numerous applications such as portable devices like cameras, phones, and computers, and in hybrid electric vehicles (HEVs), and electric vehicles (EVs). To derive the maximum output from a Li-ion battery without sacrificing safety and or durability, it is essential to accurately predict the state of the battery under all operating conditions. Unchecked faults occurring in the battery can lead to irreversible, and under extreme conditions, catastrophic damages [3, 4]. In order to avoid such conditions, it is imperative that any fault occurring in the battery be quickly detected and accurately diagnosed.

Manuscript received October 7, 2013; revised December 9, 2013, March 17, 2014 and April 30, 2014; accepted June 7, 2014.

Copyright (c) 2014 IEEE. Personal use of this material is permitted. However, permission to use this material for any other purposes must be obtained from the IEEE by sending a request to pubs-permissions@ieee.org.

A. Singh, A. Izadian, and S. Anwar are with the Purdue School of Engineering and Technology, Indianapolis, IN, 46202 USA: E-mail: aizadian@iupui.edu
Fault diagnosis in Li-ion battery has seen a growing interest among industry and academic researchers with efforts focused at detecting different battery faults using advance techniques. The use of model based fault diagnosis techniques under the paradigm of analytical redundancy have been extensively applied to the challenge of accurate fault diagnosis in Li-ion battery due to their inherent benefits of lower cost and high flexibility [5]. Observer-based fault diagnosis techniques further improve the robustness of the model-based fault diagnosis by avoiding the battery fault information loss due to faulty initial condition and unknown disturbances. The Luenberger observer (LO) [6] was applied on a string of Liion batteries using a bank of reduced order observers. LO is a suitable candidate for fault diagnosis in systems with little or no measurement noise, but with presence of noise, this setup faces inherent difficulties especially with performance variations. Kalman filters for fault detection and diagnosis in Li-ion batteries have been used [7,8], where the use of optimal filter showed strong robustness to noise. This robustness along with multiple model based fault detection method proposed here provides a framework for improved accuracy in Li-Ion battery diagnostics.

Depending on battery usage, different modeling tools can be used, namely experimental, empirical, equivalent circuit, electrochemical, and neural networks [9-11]. For real-time applications, the equivalent circuit model is best suited because of its simpler formulation and good representation of cell dynamics without high computational power demand.

The equivalent circuit model is an effective choice in system control and monitoring applications [12, 13]. The equivalent circuit parameters of some desired operations (e.g. overcharge, over-discharge, and faults) can be extracted from an offline impedance spectrum obtained through the use of AC Impedance Spectroscopy (IS) [14, 15]. IS involves passing a small amplitude of AC current through the Li-ion battery and measuring its $\mathrm{AC}$ response. From the known AC input and the measured $\mathrm{AC}$ response, the battery impedance of the desired models can be evaluated. Furthermore, inclusion of these models into the adaptive framework of the fault detection algorithm enables on-line battery fault diagnosis and condition monitoring. IS data provides the necessary parameters for the models used by the detection algorithm for real-time fault detection.

Battery impedance depends on many chemical reactions and on the state of charge (SOC), the operating temperature, and the capacity fade effects [15]. In this study, these variations are assumed to be small. The effects of non-linear elements in the equivalent circuit model are considered to be negligible, namely Warburg impedance, representing the diffusion phenomenon [16]. 


\section{IEEE TRANSACTIONS ON INDUSTRIAL ELECTRONICS}

Within the paradigm of an equivalent circuit model for a liion battery cell, the choice of circuit layout results in how closely the model depicts the cell's electrochemical phenomenon. Different equivalent circuit models exist [10, 17-19] that express resistive model to demonstrate the voltage drops from open circuit voltage (OCV) to the terminal voltage, $\mathrm{RC}$ model with series and parallel resistances and capacitors, Thevenin model, and higher order models offering various degrees of success.

The equivalent circuit used in this study is an extension to the Thevenin model; with an additional RC parallel circuit element. This model offers a better representation of electrochemical phenomenon like the distribution of reactivity at the electrode, the interfacial impedance, and the electron and ion migration resistance at a lower computational effort $[10,20]$. A higher order model with more than two RC parallel circuit elements increases the order of the system model thus resulting in higher computational expense without significantly improving the model accuracy [21]. The simplified cell model is shown in the Figure 1.

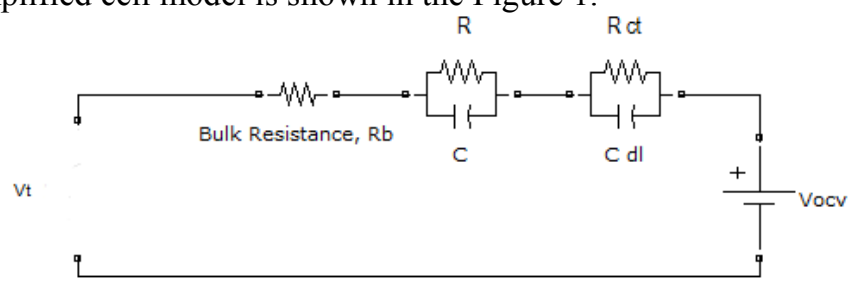

Fig. 1. Li-ion battery equivalent circuit model.

The equivalent circuit consists of resistors and capacitors where $R_{b}$ represents the bulk electrolyte resistance; the constant phase element, $C$, and resistance, $R$, are used to model the distribution of reactivity capturing the local property of the electrode, the charge transfer resistance, $R_{c t}$, and the double layer capacitance, $C_{d l}$, represent the interfacial impedance of the cell $[22,23]$. The open-circuit voltage $\left(V_{O C V}\right)$ in the equivalent circuit model has non-linear characteristics in the Li-ion battery [24, 25]. Modeling of various operating conditions, including signature faults, can be accomplished both structurally and parametrically using equivalent models. Multiples of these signature faults can provide a set of conditions to be detected in case of emergency to extend the life and reduce the stress on the battery.

The multiple model adaptive estimation (MMAE) technique [7, 26-31] has shown effective fault detection and identification techniques when the models of signature faults are obtained. Models of faults are required to generate residual signals. These signals are processed in an evaluation algorithm to generate the probability of each signature fault model at any time instance. This detects and isolates the faults. In this type of diagnosis, the generation and evaluation of residual signals directly affects the diagnosis performance [5]. Residual signals are generated by comparing the outputs of the fault models with the simulated output of the system. Mathematical models of faults can be designed to incorporate different system behaviors.

A model that closely represents the system dynamics should consider the effects of noise, shifts in parameter values, and the history of parameter variation. Nonlinear dynamics can also be used in the MMAE context using a bank of extended
Kalman filters. Like its linear counterparts, the filters represent normal and faulty system models [32, 33]. Extended Kalman filters have found extensive application in Li-ion battery equivalent circuit models for state and parameter estimation [25, 34-37]

This study aims at detecting and diagnosing over-charge (OC) and over-discharge (OD) faults in a Li-ion battery using nonlinear model representations. Both conditions are detrimental to the health of the battery, as over-charging can lead to overheating and thus vaporization of active materials. Hence, explosion and over-discharge can shorten the internal circuit of the battery cell [38]. However, if these types of failures can be detected before the system reaches failure conditions, the premature failure of the cell can be avoided.

Some of the modern battery systems come with protection circuitry, designed to protect against faults. MMAE for battery fault diagnosis can act in parallel with the protection circuitry for system redundancy. Some of the battery types have non-resettable fuses, thus rendering the battery useless after a current surge. In order for the fuse to cut the current, the fault has to have happened, and the circuit has to have experienced the fault to its full extent. A fault diagnosis is more than just an on-off switch; it provides the type of the fault occurring and predicts the changes in the circuit well ahead of time. This can be avoided with the proposed adaptive nonlinear model-based fault diagnosis. This paper is organized as follows: Section II describes the battery model, Section III describes the modelbased fault diagnosis using nonlinear models, and Section IV describes the state estimation and probabilities using extended Kalman filters. Section V is the design of the experiment, and Section VI is the discussion of the results obtained.

\section{BATTERY MODELING}

The battery cell can be modeled as a third order system using lumped resistive-capacitive electrical circuits. Among others, each of these circuit elements is a function of SOC and temperature. For this study, we assume the temperature to be constant, and only the voltage source to be a function of the SOC. Also, the aging dynamics of the system are not considered in the model. It is important to note that the signature faults occurring in the battery can be modeled to study the effects of system behavior under abnormal conditions. Faults diagnosis can also be used in effective control of the battery and to extend the battery life.

The OC failure of battery cells can be attributed to a combination of factors such as excessive temperature along with cell construction and design [39] and can lead to violent thermal runaways. The OD failures are caused due to detrimental copper plating occurring at the negative electrode which can further lead to thermal runaways under severe overdischarge [40]. Distinct parameter variation trends can be observed under both OC and OD failures. The bulk resistance, $R_{b}$, shows a more substantial increase under OC than in OD; the charge transfer resistance, $R_{c t}$, and CPE arm resistance, $R$, increase with both failure modes. This increment is more profound under OC than in OD. The double layer capacitance, $C_{d l}$, and the capacitor representing the CPE, $C$, increase rapidly with $\mathrm{OD}$, while under $\mathrm{OC}$ they show a relatively smaller change with a gradual decrease. The equivalent circuit 


\section{IEEE TRANSACTIONS ON INDUSTRIAL ELECTRONICS}

diagram is shown in Figure 1.

The open circuit voltage is represented by a voltage source, $V_{O C V}$, and is given by

$$
V_{O C V}=g(S O C)
$$

where $g$ is a non-linear function of the state of charge (SOC), given by the classic OCV-SOC curve in Figure 2.

The high-frequency response of the battery cell consists of resistance and a constant phase element (CPE) in parallel. The CPE tries to capture the distribution of reactivity at the electrodes. This phenomenon arises due to variation in surface properties. The impedance function of this pair is given by $[22,23], Z_{C P E}(\omega)=\frac{R}{1+(j \omega)^{\alpha} Q R}$, where $\alpha$ is the depression factor associated with the $\mathrm{CPE}$ and is assumed to be unity. This yields $Q=C$; hence, the CPE works as a normal capacitor $[14,22]$. Further, this high-frequency element is modeled by a single RC pair in parallel. These elements are represented by resistance, $\mathrm{R}$, and capacitance, $\mathrm{C}$, in Figure 1. Using Kirchoffs voltage law, the voltage across the capacitor, $\mathrm{C}$, is given by

$$
\dot{V}_{C}=-\frac{V_{C}}{R C}+\frac{I_{L}}{C} \text {. }
$$

The frequency response of the battery cell consists of $R_{c t}$ as the charge transfer resistance and $C_{d l}$ as the double layer capacitance element, where $I_{L}$ is the load current of the battery cell as it passes through the elements in parallel. According to the sign convention used in this study, the negative sign of $I_{L}$ represents the discharge, while the positive sign indicates the charge. The voltage across the capacitive element, $C_{d l}$, is given by

$$
V \dot{c}_{d l}=-\frac{V_{C_{d l}}}{R_{c t} C_{d l}}+\frac{I_{L}}{C_{d l}}
$$

The terminal voltage, $\mathrm{V}_{t}$, can be obtained from

$$
\mathrm{V}_{t}=I_{L} R_{b}+V_{C}+V_{C_{d l}}+V_{O C V}
$$

where $R_{b}$ is the bulk resistance. Under no load condition, $V_{O C V}$ is the open circuit voltage (OCV). The OCV is a function of the SOC [41] and is found experimentally. For this study the SOC-OCV trend, shown in Figure 2, is used. The data was recorded from a sample $\mathrm{LiFePO}_{4}$ battery cell operating at room temperature at the Energy Systems and Power Electronics Laboratory (ESPEL) at IUPUI. The data shows nonlinear behavior in a polynomial as follows:

$$
\begin{aligned}
V_{\text {OCV }}= & \\
& a_{1}\left(\operatorname{SOC}^{9}\right)+a_{2}\left(\operatorname{SOC}^{8}\right)+a_{3}\left(\operatorname{SOC}^{7}\right)+a_{4}\left(\operatorname{SOC}^{6}\right)+a_{5}\left(\operatorname{SOC}^{5}\right)+ \\
& +a_{6}\left(\operatorname{SOC}^{4}\right)+a_{7}\left(\operatorname{SOC}^{3}\right)+a_{8}\left(\operatorname{SOC}^{2}\right)+a_{9}(S O C)+a_{10}
\end{aligned}
$$

where $a_{1}=0.0385, a_{2}=-0.01936, a_{3}=-0.169, a_{4}=0.06142, a_{5}=$ $0.2328, a_{6}=-0.05715, a_{7}=-0.08321, a_{8}=0.0005257, a_{9}=0.03205$, $a_{10}=3.297$.

The $S O C$ is the ratio of the remaining capacity to the nominal capacity of the battery cell and is given by

$$
\operatorname{SOC}(t)=\operatorname{SOC}(0)+\int_{0}^{t} \frac{\eta I(\tau)}{C_{n}} d \tau
$$

where $\operatorname{SOC}(0)$ represents the initial state of charge, $C_{n}$ represents the battery cell capacity in Ampere-hour, $I$ is the charge/discharge current and the same as $I_{L}$, and $\eta$ represents the coulomb efficiency given by $\eta=\left\{\begin{array}{c}1, \text { charging } \\ 0.98 \text {, discharging }\end{array}\right.$. The discrete time version of (6) can be given as

$$
\operatorname{SOC}(k)=\operatorname{SOC}(k-1)+\frac{\eta I(k-1) \Delta t}{C_{n}} .
$$

The discrete time counterparts of (2) and (3) can be obtained using zero-order hold ( $\mathrm{ZOH})[42]$ as follows:

$$
\begin{array}{r}
V_{C}(k)=\left(e^{-\frac{\Delta t}{R C}}\right) V_{C}(k-1)+R\left[1-\left(e^{-\frac{\Delta t}{R C}}\right)\right] I(k-1), \\
V_{C_{d l}}(k)=\left(e^{-\frac{\Delta t}{R_{c t} C_{d l}}}\right) V_{C_{c l}}(k-1)+R_{c t}\left[1-\left(e^{-\frac{\Delta t}{R_{c t} C_{d l}}}\right)\right] I(k-1) .
\end{array}
$$

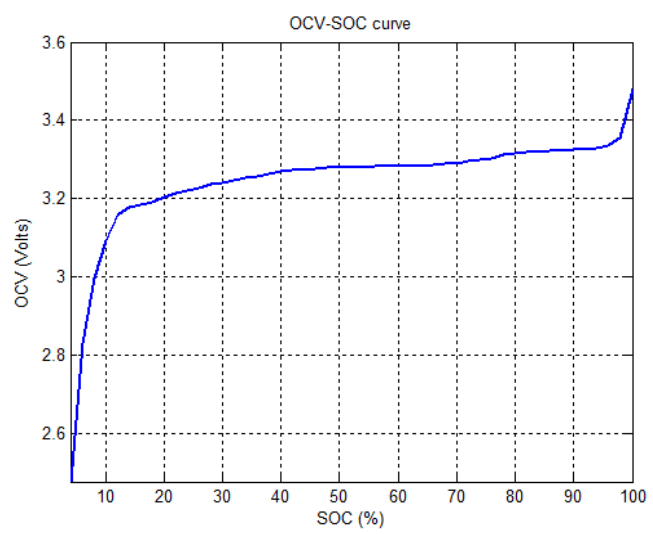

Fig. 2. Experimental OCV-SOC curve for $\mathrm{LiFePO} 4$ battery cell under testing.

The nonlinear battery model has the following state variables $x=\left[\begin{array}{llll}S O C & V_{C} & V_{C_{d l}}\end{array}\right]^{T}$ presented by

$$
\begin{aligned}
& x(k)=f\left(x_{k-1}, I_{k-1}\right)+w_{k-1} \\
& z(k)=h\left(x_{k}, I_{k}\right)+v_{k},
\end{aligned}
$$

where $f$ and $h$ are the continuously differentiable nonlinear functions, while $w$ is the input noise with zero mean and a variance of

$$
E\left\{w_{n}[l] w_{n}{ }^{T}[m]\right\}=\left\{\begin{array}{ll}
Q, & l=m \\
0, & l \neq m
\end{array},\right.
$$

and $v$ is the measurement noise, independent from $w$, with zero mean value as

$$
E\left\{v_{n}[l] v_{n}{ }^{T}[m]\right\}= \begin{cases}R, & l=m \\ 0, & l \neq m\end{cases}
$$

In this formulation, $Q$ and $R$ are the process and measurement noise variances, respectively. The process and measurement white Gaussian noise is generated using the polar method [43]. From (7), (8), and (9) the function $f$ is given by

$$
\left.\begin{array}{l}
f(k-1)= \\
\operatorname{SOC}(k-1)+\frac{\eta \Delta \mathrm{t}(\mathrm{k}-1)}{C_{n}} \\
\left(e^{-\frac{\Delta t}{R C}}\right) V_{C}(k-1)+R\left[1-\left(e^{-\frac{\Delta t}{R C}}\right)\right] I(k-1) \\
\left(e^{-\frac{\Delta t}{R_{c t} C_{d l}}}\right) V_{C_{d l}}(k-1)+R_{c t}\left[1-\left(e^{-\frac{\Delta t}{R_{c t} C_{d l}}}\right)\right] I(k-1)
\end{array}\right]
$$

From (4) and (5) the function $h$ is given by

$$
\begin{aligned}
h(k)= & I(k) R_{b}+V_{C}+V_{C_{d l}}+ \\
& a_{1}\left(S O C^{9}\right)+a_{2}\left(S^{8} C^{8}\right)+a_{3}\left(S O C^{7}\right)+a_{4}\left(S O C^{6}\right)+a_{5}\left(S O C^{5}\right)+
\end{aligned}
$$

The lumped electrical elements and their associated scalar values represent the model of the battery cell at any given time. Considering the different values for the electrical elements $R_{b}, R, C, R_{c t}$, and $C_{d l} ; n$ distinct models can be obtained, each representing a signature fault or the health of the battery cell.

With varying battery conditions and subsequent signature 


\section{IEEE TRANSACTIONS ON INDUSTRIAL ELECTRONICS}

faults, the OCV-SOC trend for the battery shifts. This shift can be modeled in two approaches, 1) updating the coefficients in (5) and 2) incorporating the parameter variations in the equivalent circuit parameters. The later leads to greater emphasis being applied on the fault information carrying equivalent circuit parameter values and polarization voltages rather than the OCV-SOC variation characteristics.

\section{ADAPTIVE NONLINEAR MODEL-BASED DiAGNOSIS}

The model-based fault diagnosis structure used in this paper is shown in Figure 3.

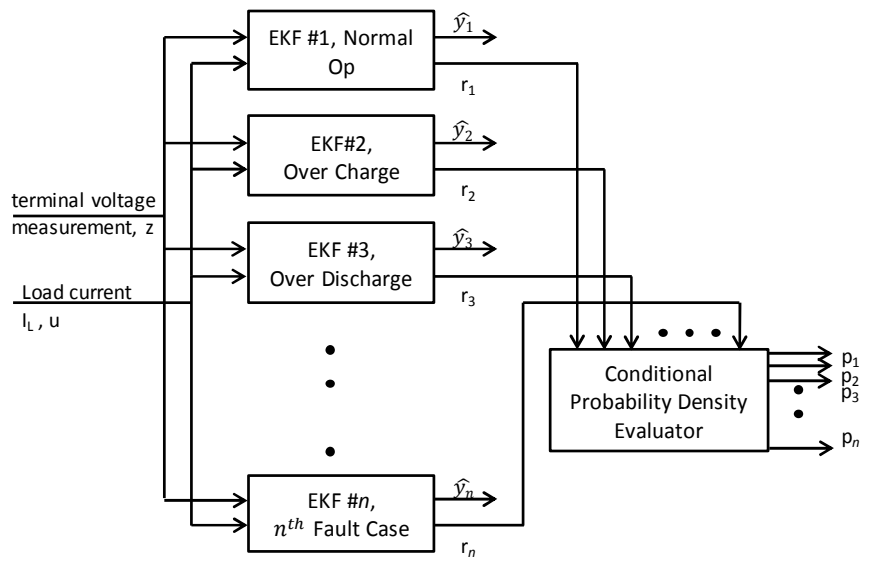

Fig. 3. Multiple-model residual generation and probability evaluation.

As described earlier, several models can be designed to accurately represent $n$ signature faults. The same input signal that drives the actual system is required to excite all models simultaneously. Therefore, each fault-representing model generates an exclusive output. If there is a fault in the system matching any of the signature fault models, the actual system's output will match with the output of one of the fault representing models. Therefore, the difference between their outputs, the residual signal, becomes a zero mean value signal.

The existence of noise in the actual settings results in fault information loss; therefore, the fault diagnosis becomes insensitive to small parameter variations. Several modeling techniques have been introduced to estimate the output of the fault models in a noisy environment.

In the next section, extended Kalman filters [25, 34, 41, 44, 45] are introduced and used to generate residual signals. The generated residual signals are evaluated in a probability-based approach to indicate the probability of each fault that may have occurred.

\section{State Estimation AND ProbabiLities}

\section{A. Extended Kalman Filter Design}

An extended Kalman filter is used for estimating the states of the nonlinear systems by linearization around the current mean and covariance. The discrete extended Kalman filter aims to estimate the state of the system given by (10)-(13). The extended Kalman prediction equations are given by [44, 45]:

$$
\begin{aligned}
& \hat{x}_{k \mid k-1}=f\left(\hat{x}_{k-1 \mid k-1}, I_{k-1}\right) \\
& P_{k \mid k-1}=F_{k-1} P_{k-1 \mid k-1} F_{k-1}{ }^{T}+Q_{k-1} .
\end{aligned}
$$

The recursive equations are given by

$$
\begin{aligned}
& K_{k}=P_{k \mid k-1} H_{k}{ }^{T}\left(H_{k} P_{k \mid k-1} H_{k}{ }^{T}+R_{k}\right)^{-1} \\
& \hat{x}_{k \mid k}=\hat{x}_{k \mid k-1}+K_{k}\left(z_{k}-h\left(\hat{x}_{k \mid k-1}\right)\right), \\
& P_{k \mid k}=\left(I-K_{k} H_{k}\right) P_{k \mid k-1}
\end{aligned}
$$

where $\hat{x}_{k \mid k-1}$ represents the predicted state based on the function, $f$, evaluated at the estimated state and inputs available at sample $k-1 . P_{k \mid k-1}$ is the predicted covariance estimate; $K_{k}$ is the Kalman gain; $\hat{x}_{k \mid k}$ is the updated state estimate; and $P_{k \mid k}$ is the updated covariance estimate. The state transition matrix $F_{k-1}$ and observation matrix $H_{k}$ are given as $F_{k-1}=\left.\frac{\partial f}{\partial x}\right|_{\hat{x}_{k-1 \mid k-1}, I_{k-1}}$ and $H_{k}=\left.\frac{\partial h}{\partial x}\right|_{\hat{x}_{k \mid k-1}, I_{k-1}}$. The estimated output is obtained from

$$
\hat{y}_{k}=h\left(\hat{x}_{k \mid k}, I_{k}\right) \text {. }
$$

To obtain the residual signals of the models as shown in Figure 3, the estimated output signal from the signature fault cases is subtracted from the measured output of the system. This is obtained from the following equation:

$$
r=z(k)-\hat{y}_{k} .
$$

\section{B. Multiple-Model Adaptive Estimation Technique}

In the multiple model adaptive estimation (MMAE) technique, as shown in Figure 3, several models run in parallel while excited with a similar input signal as that of the actual system generating residuals. To evaluate and extract the fault information from the residual signals, an evaluation algorithm should continuously monitor the residual signal variations. If the output of any model matches the output of the actual system and makes the mean value of the residual signal zero, then the covariance of that signal evaluated at each sample can be given by [27-29]:

$$
\psi_{n, k}=C_{n, k} P_{n, k \mid k} C_{n, k}^{T}+R,
$$

where $C_{n, k}=\left.\frac{\partial h}{\partial x}\right|_{\hat{x}_{k \mid k}}$ is the linearized output vector evaluated at the current estimated state. In this paper, probability-based residual-signal evaluation was applied to the residual signals. Conditional probability density functions of the $n^{\text {th }}$ model considering the history of measurement $Z\left(t_{i-1}\right)=$ $\left[z^{T}\left(t_{1}\right) \ldots z^{T}\left(t_{i-1}\right)\right]$ are expressed as [26-28]:

$$
f_{Z(k) \mid a, Z(k-1)}\left(\mathbf{z}_{k} \mid \boldsymbol{a}_{n}, Z_{k-1}\right)=\beta_{n} \exp (\circ),
$$

where

$$
\beta_{n}=\frac{1}{(2 \pi)^{l / 2}\left|\psi_{n}(k)\right|^{1 / 2}},
$$

$l=1$ is the measurement dimension, and

$$
\text { (o) }=-\frac{1}{2} r_{n}{ }^{T}(k) \psi_{n, k}{ }^{-1} r_{n}(k)
$$

where $r_{n}$ is the residual signal for the $n^{\text {th }}$ model.

The conditional probability evaluation of the $n^{\text {th }}$ sub-system is given [26-28] by

$$
p_{n}(k)=\frac{f_{Z(k) \mid a, Z(k-1)}\left(\boldsymbol{z}_{k} \mid \boldsymbol{a}_{n}, Z_{k-1}\right) p_{n}(k-1)}{\sum_{j=1}^{n} f_{Z(k) \mid a, Z(k-1)}\left(\mathbf{z}_{k} \mid \boldsymbol{a}_{j}, Z_{k-1}\right) p_{j}(k-1)},
$$

where $p_{j}$ is the conditional probability of $j^{\text {th }}$ model, $j=1,2, \ldots$, $\mathrm{n}$. The conditional probability density functions require $a$ priori samples to compute the current values and are normalized over a complete sum of conditional probabilities of all systems [26-28]. The largest conditional probability among all can be used as an indicator of fault in the systems. In some applications, where probabilities change rapidly and make the output of the system unpredictable, the output should 
IEEE TRANSACTIONS ON INDUSTRIAL ELECTRONICS

then be compared with a threshold [29].

\section{DESIGN OF EXPERIMENT}

As mentioned earlier, faults in a battery cell can be defined by substantial parameter variations that result in sensible changes to the battery's operation. This study primarily focuses on overcharge (OC) and over-discharge (OD) faults in a battery cell. The OC condition is achieved by cycling the battery with $120 \%$ charge and $100 \%$ nominal discharge at a suitable current rate. The OD condition, on the other hand is realized by cycling the battery with $100 \%$ nominal charge and $120 \%$ discharge at a suitable current rate. When considering OC and OD battery cell fault types, each of the system parameters such as bulk resistance, charge transfer resistance and double layer capacitance show a particular trend in parameter variation. This trend can be seen in the impedance spectroscopy results in Tables I and II.

The AC impedance spectroscopy was carried out using an 8-channel Solartron 1470E Multistat device. The technique involved applying a frequency sweep of $1 \mathrm{MHz}$ to $0.01 \mathrm{~Hz}$ with the amplitude of $5 \mathrm{mV}$ to the battery system. The test setup involved the use of a PC that ran application software to control the $\mathrm{AC}$ impedance spectroscopy equipment, store and process the data such as LEVM/LEVMW complex nonlinear least squares (CNLS) fitting program [46], and display the graphical results. After fixing the equivalent circuit model and using the LEVM software interface, the program fits the desired cell model to the measured cell impedance [23].

A A123 $18650 \mathrm{LiFePO}_{4}$ battery cell was used in the experiments [47]. Tables I and II illustrate the impedance spectroscopy results for the selected circuit parameters fitted to the impedance curve when the battery cell was under different degree of fault conditions. These impedance measurements were carried out at the end of appropriate cycles with the OCV of 2.8 Volts.

Since OC resulted in gradual parameter variations, the battery had to be gone under more number of OC test cycles, as shown in Table I. The AC impedance spectroscopy was performed at the end of cycles $1,5,10,12,15$ and 18 to identify the OC battery cell parameter values. In Table II, a new battery cell was subjected to continuous cycles of OD. The AC impedance spectroscopy was performed after cycles $1,2,3,4,5$, and 6 . From Table I, the parameter values corresponding to $18^{\text {th }}$ cycle were used in formulating the $\mathrm{OC}$ fault model.

The OD fault model was based on the $6^{\text {th }}$ cycle parameter values in Table II. The healthy battery model parameters were selected from the first cycle of the OD impedance spectroscopy data in Table II. The impedance spectroscopy results are in fact functions of temperature, SOC and aging. However, in this study, only the effect of SCO was considered.

TABLE I.

IMPEDANCE SPECTROSCOPY DATA IN OVER CHARGE

\section{Overcharge}

$\begin{array}{cccccc}\text { Cycle } & \mathbf{R}_{\mathbf{0}}(\boldsymbol{\Omega}) & \mathbf{C}(\mathbf{F}) & \mathbf{R}(\mathbf{\Omega}) & \mathbf{C}_{\text {dl }}(\mathbf{F}) & \mathbf{R}_{\text {ct }}(\mathbf{\Omega}) \\ \mathbf{1} & 0.0771 & 0.0265 & 0.0156 & 0.4177 & 0.0282 \\ \mathbf{5} & 0.2433 & 0.0041 & 0.0369 & 0.2463 & 0.0329 \\ \mathbf{1 0} & 0.1395 & 0.0018 & 0.0720 & 0.1651 & 0.0376\end{array}$

$\begin{array}{llllll}\mathbf{1 2} & 0.1387 & 0.0012 & 0.1429 & 0.1007 & 0.0500 \\ \mathbf{1 5} & 0.2865 & 0.0010 & 0.2571 & 0.0589 & 0.0763 \\ \mathbf{1 8} & 0.1661 & 0.0007 & 0.4907 & 0.0140 & 0.1833\end{array}$

TABLE II.

IMPEDANCE SPECTROSCOPY DATA IN OVER DISCHARGE

\begin{tabular}{cccccc}
\multicolumn{7}{c}{ Over-discharge } \\
Cycle & $\mathbf{R}_{\mathbf{0}}(\mathbf{\Omega})$ & $\mathbf{C}(\mathbf{F})$ & $\mathbf{R}(\mathbf{\Omega})$ & $\mathbf{C}_{\text {dl }}(\mathbf{F})$ & $\mathbf{R}_{\text {ct }}(\mathbf{\Omega})$ \\
$\mathbf{1}$ & 0.0503 & 0.1922 & 0.0051 & 0.8213 & 0.0126 \\
$\mathbf{2}$ & 0.0566 & 0.2623 & 0.0045 & 2.6470 & 0.0098 \\
$\mathbf{3}$ & 0.0578 & 0.2669 & 0.0055 & 3.2500 & 0.0123 \\
$\mathbf{4}$ & 0.0594 & 0.4379 & 0.0053 & 4.2580 & 0.0126 \\
$\mathbf{5}$ & 0.0569 & 0.4067 & 0.0056 & 4.3360 & 0.0112 \\
$\mathbf{6}$ & 0.0623 & 0.2590 & 0.0054 & 2.9430 & 0.0081
\end{tabular}

The load current applied on the $18650 \mathrm{LiFePO}_{4}$ battery cell is based on the UDDS drive cycle for an electric vehicle which has been scaled appropriately to match the capacity of one battery cell, obtained using AUTONOMIE [48]. The duration of the cycle considered for the study is 71 seconds, as seen in Figure 4; the battery model response to this current can be seen in Figure 5. Battery cells having higher capacity will have higher amplitude of the load current. While the load current profile simulates the actual working conditions of the system, the resulting fault probabilities depend more on the zero average residual signal than the magnitude of the load current.

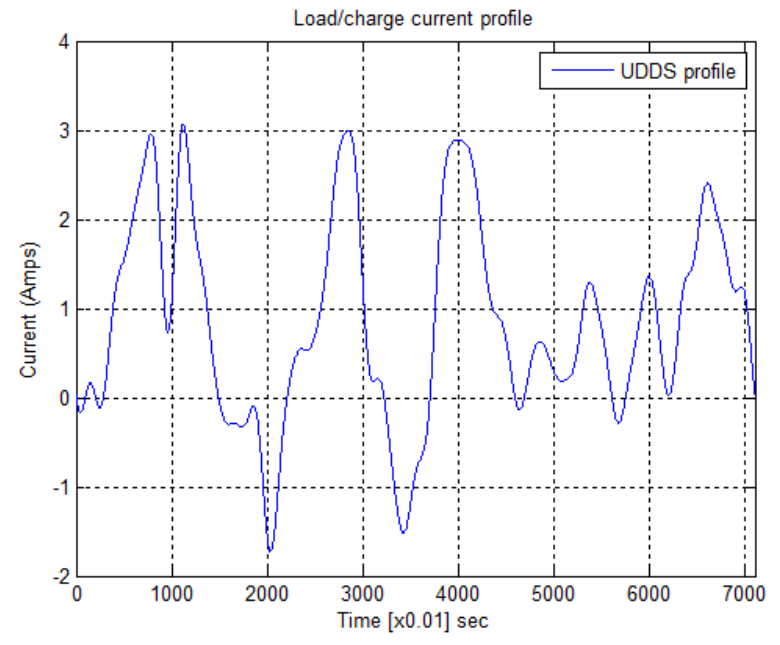

Fig. 4. Battery cell current profile.

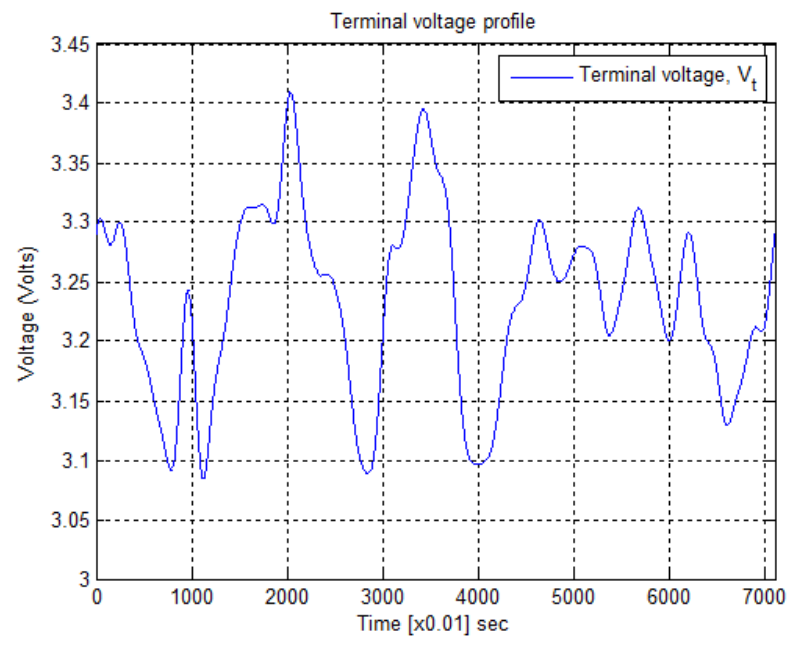




\section{IEEE TRANSACTIONS ON INDUSTRIAL ELECTRONICS}

Fig. 5. Simulated battery cell terminal voltage when UDDS load current is applied.

Each of the models experiences the same input load current and terminal voltage measurements. Based on these inputs to extended Kalman filters, the estimated terminal voltage and residuals are calculated. The initial state of the system is considered to be $\left[\begin{array}{lll}0.7 & 0 & 0\end{array}\right]^{T}$, which implies $70 \%$ SOC and zero polarization voltages.

In each stage of fault diagnosis, proper circuit parameter values are used to indicate the true effects of over-charging or over-discharging. Therefore, the fault scenario demonstrated the deteriorated battery under operation in the UDDS driving cycle. Terminal voltage along with input current signals were used to indicate in real-time if the cell was in health, OC or OD condition.

\section{DIAGNOSIS PERFORMANCE}

\section{A. Fault Diagnosis}

To simulate the effectiveness of the fault diagnosis technique, parameter variation is induced in the measurement to represent consecutive fault cases. The simulation is run for a total of 7100 samples, from which healthy operation is simulated for the first 1775, and the last 1775 represent identical start and end conditions. This setup helps to check the effectiveness of the algorithm to de-latch itself from a diagnosed fault case [29]. The faults are injected based on the following steps:

The total simulation is divided into four equal parts which occur consecutively:

1. For the first 1775 samples, healthy battery cell operation.

2. For the next 1775 samples, OC fault condition.

3. For the next 1775 samples, OD fault condition.

4. For the remaining 1775 samples, a return to healthy battery cell operation.

It is assumed that only one type of fault can occur in the system at a given point in time. The conditional probability evaluator block evaluates the probability of occurrence of a particular fault. At each time step, the probabilities are evaluated to obtain a value between 0 and 1 , where 0 means no fault and 1 means a definite fault case.

\section{1) Unbounded SOC Estimation}

In this case, the upper and lower bound parameters of state of charge estimation were not limited. From Figure 6, three probabilities can be observed, where $P_{n}$ represents the probability of the healthy operation of the cell, while $P_{o c}$ and $P_{o d}$ indicate the probability of the OC and the OD fault occurrence respectively.

The resulting probabilities shown in Figure 6 illustrate random behaviors and do not indicate the faults as expected according to the fault scenario discussed in the sections above. This behavior of fault probabilities can be attributed to inaccurate filter output, which, in turn, leads to unexpected residual change. The probabilities along with the residuals can be seen in Figure 7.

As the figure illustrates, the signature faults could not be accurately detected while the probability associated with each model changed frequently. This resulted in the Open Circuit Voltage to be extensively over- or underestimated. To resolve this issue, the upper and lower limits of the SOC were bounded. The polarization voltage for the fault models captures the shift in the electrochemical properties of the battery cell once the OC or OD has occurred.

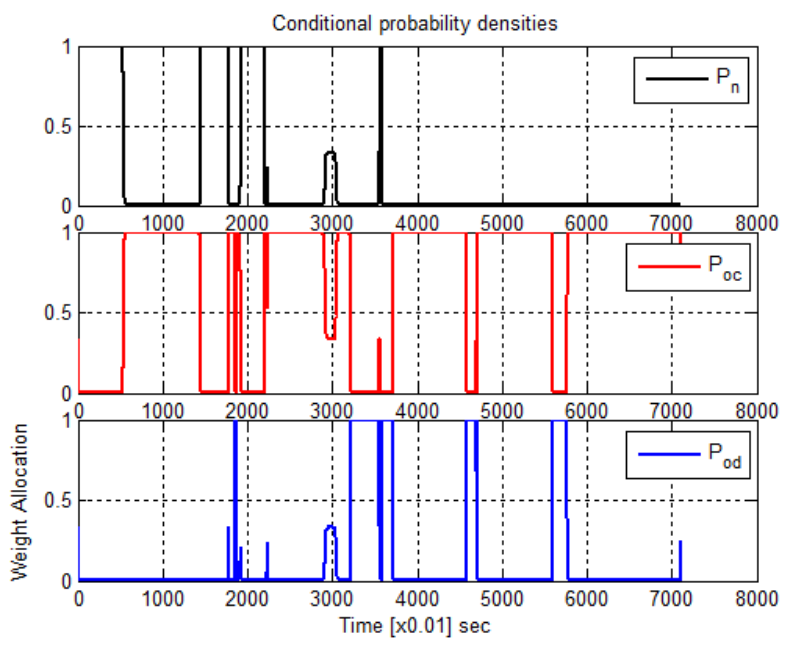

Fig. 6. Conditional probability density evaluated for normal operation, OC and OD faults.
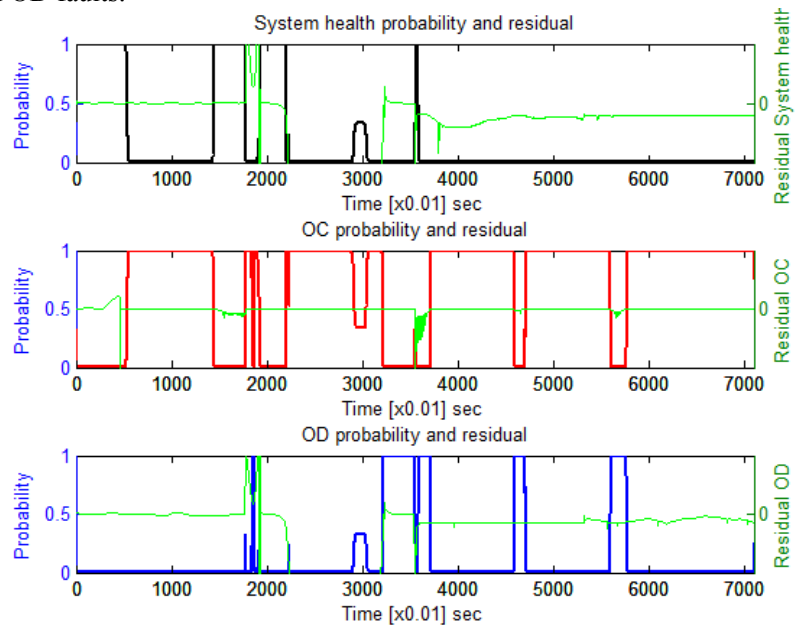

Fig. 7. Conditional probability density and residuals evaluated for normal operation and OC and OD faults.

\section{2) Bounded SOC Estimation}

The error in terminal voltage estimation was traced to the unbounded SOC estimation. The lower bound on the $S O C$ mimics the physical constraint on the battery to be depleted down to zero percent but not to negative voltages. An upper bound on SOC keeps the Open Circuit Voltage under permissible limits, thus weighing the polarization voltages more. The polarization voltages are the voltage drops across the constant phase element, $C$, and the double layer capacitance, $C_{d l}$, elements in the equivalent circuit. Upper and lower bounding the SOC to 1 and 0 respectively resulted in a more accurate diagnosis using MMAE. The resulting terminal voltages and probabilities are shown in Figure 8 and Figure 9, respectively.

In Figure 8, during the first and last 1775 samples, the normal case, $\hat{y}_{n}$, matched with simulated measurement while 


\section{IEEE TRANSACTIONS ON INDUSTRIAL ELECTRONICS}

the overcharge, $\hat{y}_{o c}$, and over-discharge, $\hat{y}_{o d}$, showed deviation from the simulated measurement. From 1776 to 3550 , the $\hat{y}_{o c}$ matched with the simulated measurement, while both $\hat{y}_{n}$ and $\hat{y}_{o d}$ showed deviation from the simulated measurement. Finally, from 3551 to 5325 , the estimated terminal voltage from the over-discharge filter, $\hat{y}_{o d}$ matched closely with the simulated measurement, while $\hat{y}_{o c}$ showed deviation and $\hat{y}_{n}$ showed a relatively large difference from the simulated measurement. Overall, the residual signal of the signature faults for the period of the scenario matching resulted in lower values. This can assure higher probabilities associated with the low residual signature faults.

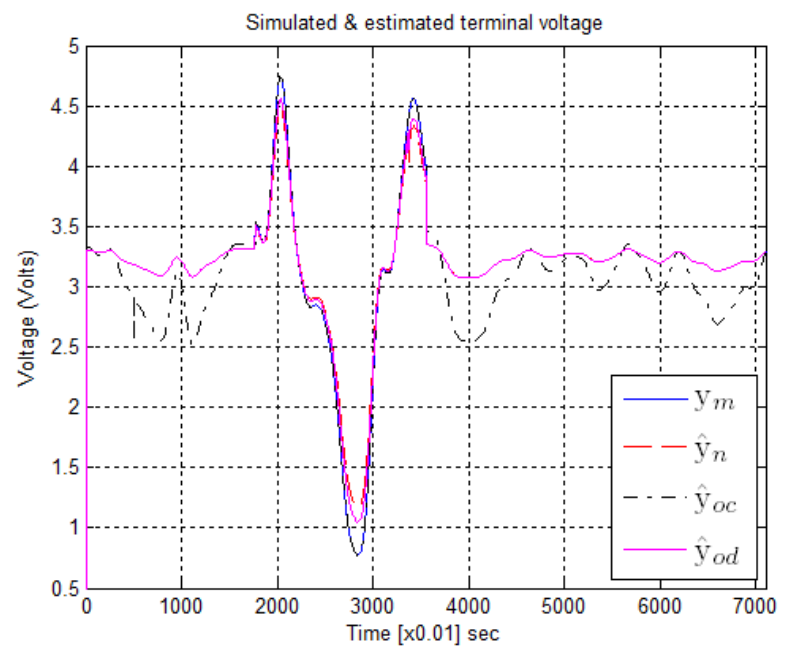

Fig. 8. Terminal voltage with bounded SOC: simulated measurement, normal, and $\mathrm{OC}$ and $\mathrm{OD}$.

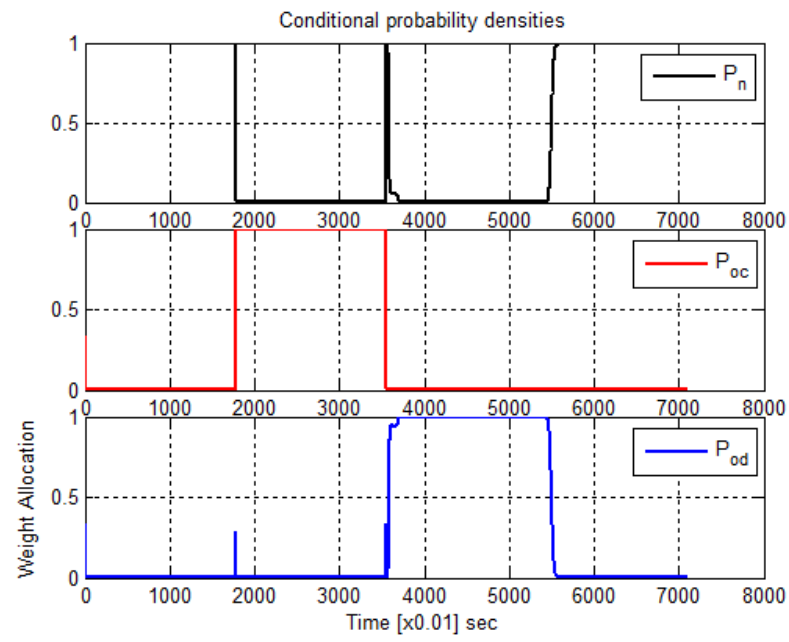

Fig. 9. Conditional probability density evaluated for normal operation and OC and OD faults with bounded SOC.

The OC fault was injected at 1775, where it can be observed in Figure 9 that the healthy battery operation probability, $P_{n}$, reached zero, indicating the presence of a fault or the nonexistence of a healthy condition. The fault type was indicated by the probability, $P_{o c}$, when it transitioned from 0 to 1 , indicating an OC fault. At the 3550 data sample, probability $P_{o d}$ was transitioned to 1 , while $P_{o c}$ reached zero, representing an OD fault. The healthy cell operation was indicated at 5325 when probability $P_{n}$ transitioned to 1 and both $P_{o c}$ and $P_{o d}$ reached 0 . The injected faults in the system were accurately detected and diagnosed using the nonlinear adaptive model-based fault diagnosis as seen in Figure 10.

At each of the simulated fault regions, the estimation from the EKF matched closely to the simulated measurement and resulted in zero mean residual, as is demonstrated in Figure 9.
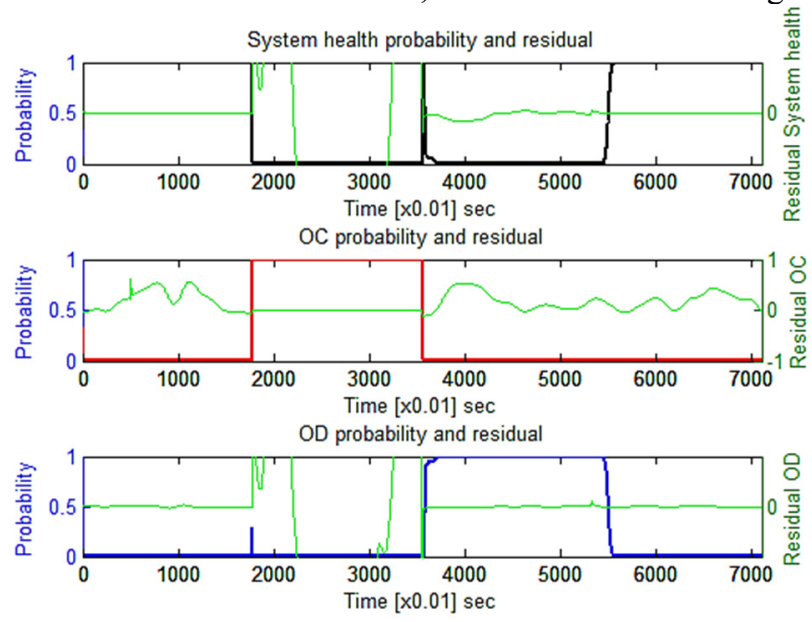

Fig. 10. Conditional probability density and residuals evaluated for normal operation and OC and OD faults with bounded SOC.

The high weight on the polarization voltages because of $S O C$ bounding resulted in accurate fault detection and diagnosis, as can be seen in Figure 10. The $S O C$ variation is demonstrated in Figure 11.

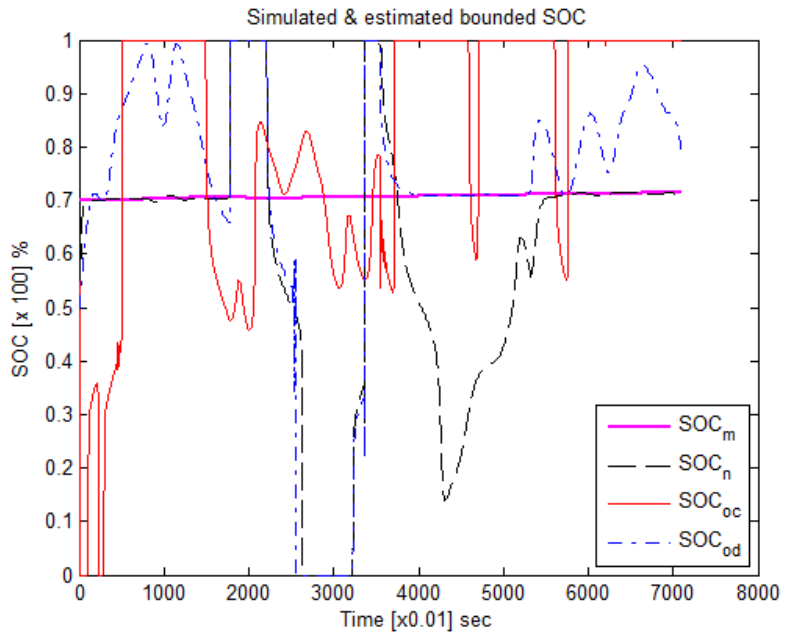

Fig. 11. Bounded SOC variation for normal operation and OC and OD faults.

In Figure 11, during the first and last 1775 samples, $S O C_{n}$ for normal operation matched with the simulated $S O C_{m}$, while in the same sample times, the OC and OD SOCs deviated from $S O C_{m}$. A closer look at this region is shown in Figure 12, where gradually increasing $S O C_{m}$ is available through Coulomb considering the cell capacity and the UUDS demanded battery current profile. 


\section{IEEE TRANSACTIONS ON INDUSTRIAL ELECTRONICS}

From sample points 1776 to 3550 , the $S O C_{o c}$ for $\mathrm{OC}$ operation matched with $S O C_{m}$ while $S O C_{n}$ and $S O C_{o d}$ showed a relatively large deviation. It is important to note that an overall match offered by an SOC estimation is more important than absolute matching od SOCs. Finally, from sample points 3551 to $5325, S O C_{o d}$ matched with $S O C_{m}$ while $S O C_{n}$ and $S O C_{o c}$ deviated from $S O C_{m}$.

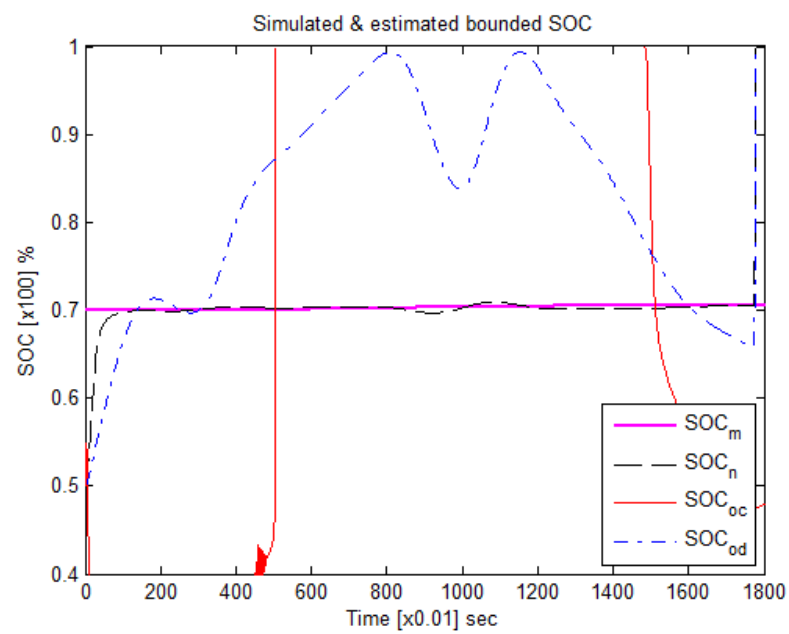

Fig. 12. Bounded SOC variation: zero to 1800 samples.

The simulated results indicate the effectiveness of Li-ion battery fault detection based on a nonlinear battery model and the utilization of an extended Kalman filter and multiple model adaptive estimation schemes. The desired signature faults of distinct conditions, even as combined parameter variations, could be modeled using an extended Kalman filter. Signature faults could be picked in MMAE by generating and mapping models to higher probability values.

It was also demonstrated that limiting SOC improves the fault diagnosis performance by better estimating the terminal voltage under various conditions.

The use of MMAE for li-ion battery fault detection offers tremendous advantages over traditional set point comparison methods. The proposed method provides better insight into the state of the battery such as accurate SOC estimation at any given time.

Before a particular set point is reached, this method is capable of providing valuable information regarding the trend in parameter variation. The proposed method also ensures that false alarms are not triggered as it takes into consideration more factors than just voltage and SOC. The effectiveness of the proposed technique can be further enhanced by adding the effect of temperature or ageing into the battery model. Model based fault diagnosis along with optimal filters offers good robustness against noise and faulty initial conditions.

\section{CONCLUSION}

This paper illustrated how an adaptive model-based technique was used to diagnosis over-charging and overdischarging faults in Li-ion batteries. Nonlinear characteristics of these battery cells were modeled using Extended Kalman filters to define signature fault models. This technique provided an innovative approach in using off-line recorded battery cell parameters in a real-time fault diagnosis. The proposed algorithm can be added to the existing microcontroller of hybrid electric or pure electric vehicles and can provide a more robust and accurate diagnosis and condition monitoring of a Li-Ion cell. This fault diagnosis approach advances the battery protection field by using multiple models of the equivalent circuit to emulate the fault conditions and using system identification tools to estimate the model parameter for these fault conditions. The proposed fault diagnosis methodology can be further developed for other types of batteries with appropriate equivalent circuit models and proper open circuit voltage versus state of charge relationships. Effectiveness of the proposed fault diagnosis algorithms was experimentally demonstrated in a laboratory setting.

\section{ACKNOWLEDGMENT}

The authors would like to thank Dr. J. Xie and Dr. Yadong for $18650 \mathrm{LiFePO}_{4}$ impedance spectroscopy data.

\section{REFERENCES}

[1]J. M. Tarascon and M. Armand, "Issues and challenges facing rechargeable lithium batteries," Nature, vol. 414, pp. 359-67, Nov 152001.

[2]T. G. Goonan, "Lithium use in batteries," vol. Circular 1371, 14 p., U. S. G. Survey, Ed., ed, 2012.

[3]J. Zhang and J. Lee, "A review on prognostics and health monitoring of Liion battery," Journal of Power Sources, vol. 196, pp. 6007-6014, 8/1/ 2011.

[4]K. Goebel, B. Saha, A. Saxena, J. Celaya, and J. Christophersen, "Prognostics in Battery Health Management," IEEE Instrumentation \& Measurement Magazine, vol. 11, pp. 33-40, 2008.

[5]S. X. Ding, Model-based Fault Diagnosis Techniques: Design Schemes, Algorithms, and Tools: Springer Publishing Company, Incorporated, 2008.

[6]W. Chen, W. T. Chen, M. Saif, M. F. Li, and H. Wu, "Simultaneous Fault Isolation and Estimation of Lithium-Ion Batteries via Synthesized Design of Luenberger and Learning Observers," IEEE Trans. on Control Systems Technology, , vol. PP, pp. 1-1, 2013.

[7]A. Singh, A. Izadian, and S. Anwar, "Fault Diagnosis of Li-Ion Batteries Using Multiple-Model Adaptive Estimation," presented at the IEEE Ind. Electron., IECON 2013 - 39th Annual Conference on, Vienna, Austria, 2013.

[8]A. Singh, A. Izadian, and S. Anwar, "Nonlinear Model Based Fault Detection of Lithium Ion Battery Using Multiple Model Adaptive Estimation," presented at the 19th IFAC World Congress, Cape Town, South Africa, 2014.

[9] O. Tremblay, L. A. Dessaint, and A. I. Dekkiche, "A Generic Battery Model for the Dynamic Simulation of Hybrid Electric Vehicles," in IEEE Vehicle Power and Propulsion Conference, 2007. VPPC 2007., 2007, pp. 284-289.

[10] H. He, R. Xiong, and J. Fan, "Evaluation of lithium-ion battery equivalent circuit models for state of charge estimation by an experimental approach," Energies, vol. 4, pp. 582-598, 2011.

[11] M. Charkhgard and M. Farrokhi, "State-of-Charge Estimation for Lithium-Ion Batteries Using Neural Networks and EKF," IEEE Trans. on Ind. Electron., vol. 57, pp. 4178-4187, 2010.

[12] T. Xidong, M. Xiaofeng, L. Jian, and B. Koch, "Li-ion battery parameter estimation for state of charge," in American Control Conference (ACC), 2011, 2011, pp. 941-946.

[13] A. H. Ranjbar, A. Banaei, A. Khoobroo, and B. Fahimi, "Online Estimation of State of Charge in Li-Ion Batteries Using Impulse Response Concept," IEEE Trans. on Smart Grid, vol. 3, pp. 360-367, 2012.

[14] S. Buller, "Impedance-Based Simulation Models for Energy Storage Devices in Advanced Automotive Power Systems," Phd, Institute for Power Electronics and Electrical Drives, RWTH Aachen University, Germany, 2003.

[15] S. Buller, M. Thele, R. W. A. A. De Doncker, and E. Karden, "Impedance-based simulation models of supercapacitors and $\mathrm{Li}$-ion 


\section{IEEE TRANSACTIONS ON INDUSTRIAL ELECTRONICS}

batteries for power electronic applications," IEEE Trans. on Ind. Applications, vol. 41, pp. 742-747, 2005.

[16] T. C. Kaypmaz and R. N. Tuncay, "An advanced cell model for diagnosing faults in operation of Li-ion Polymer batteries," in IEEE Vehicle Power and Propulsion Conference (VPPC), 2011, 2011, pp. 1-5.

[17] Y.-H. Chiang, W.-Y. Sean, and J.-C. Ke, "Online estimation of internal resistance and open-circuit voltage of lithium-ion batteries in electric vehicles," Journal of Power Sources, vol. 196, pp. 3921-3932, 2011.

[18] A. Hentunen, T. Lehmuspelto, and J. Suomela, "Electrical battery model for dynamic simulations of hybrid electric vehicles," in IEEE Vehicle Power and Propulsion Conference (VPPC), 2011, 2011, pp. 1-6.

[19] R. C. Kroeze and P. T. Krein, "Electrical battery model for use in dynamic electric vehicle simulations," in IEEE Power Electronics Specialists Conference, 2008. PESC 2008., 2008, pp. 1336-1342.

[20] X. Tang, X. Mao, J. Lin, and B. Koch, "Li-ion battery parameter estimation for state of charge," in American Control Conference (ACC), 2011, 2011, pp. 941-946.

[21] T. Huria, M. Ceraolo, J. Gazzarri, and R. Jackey, "High fidelity electrical model with thermal dependence for characterization and simulation of high power lithium battery cells," in IEEE International Conferencen on Electric Vehicle Conference (IEVC), 2012, pp. 1-8.

[22] M. E. Orazem and B. Tribollet, Electrochemical Impedance Spectroscopy: John Wiley \& Sons, Inc., 2008.

[23] E. Barsoukov and J. R. Macdonald, Impedance Spectroscopy: Theory, Experiment, and Applications: Wiley, 2005.

[24] C. Min and G. A. Rincon-Mora, "Accurate electrical battery model capable of predicting runtime and I-V performance," IEEE Trans. on Energy Conversion, , vol. 21, pp. 504-511, 2006.

[25] Z. Xiang, Z. Bingzhan, Z. Han, S. Weixiang, and A. Kapoor, "State of charge estimation based on improved Li-ion battery model using extended Kalman filter," in the $8^{\text {th }}$ IEEE Conference on Industrial Electronics and Applications (ICIEA), 2013, pp. 607-612.

[26] P. Eide and P. Maybeck, "Implementation and demonstration of a multiple model adaptive estimation failure detection system for the F-16," in Proceedings of the 34th IEEE Conference Decision and Control, 1995., on, 1995, pp. 1873-1878 vol.2.

[27] P. Eide and P. Maybeck, "An MMAE failure detection system for the F16," IEEE Trans. on Aerospace and Electronic Systems, , vol. 32, pp. 1125-1136, 1996.

[28] P. D. Hanlon and P. S. Maybeck, "Multiple-model adaptive estimation using a residual correlation Kalman filter bank," IEEE Trans. on Aerospace and Electronic Systems , vol. 36, pp. 393-406, 2000.

[29] A. Izadian and P. Famouri, "Fault Diagnosis of MEMS Lateral Comb Resonators Using Multiple-Model Adaptive Estimators," IEEE Trans. on Control Systems Technology, vol. 18, pp. 1233-1240, 2010.

[30] A. Izadian, P. Khayyer, and P. Famouri, "Fault Diagnosis of TimeVarying Parameter Systems With Application in MEMS LCRs," IEEE Trans. on Ind. Electron., vol. 56, pp. 973-978, 2009.

[31] A. Izadian, "Self-Tuning Fault Diagnosis of MEMS," International Federation on Automatic Control, Journal of Mechatronics, vol. 23, issue 8, pp. 1094-1099, Dec. 2013.

[32] J. Marzat, H. Piet-Lahanier, F. Damongeot, and E. Walter, "Modelbased fault diagnosis for aerospace systems: A survey," Proceedings of the Institution of Mechanical Engineers, Part G: Journal of Aerospace Engineering, vol. 226, pp. 1329-1360, 2012.

[33] D. Rupp, G. Ducard, E. Shafai, and H. P. Geering, "Extended Multiple Model Adaptive Estimation for the Detection of Sensor and Actuator Faults," in Decision and Control, 2005 and 2005 European Control Conference. CDC-ECC '05. 44th IEEE Conference on, 2005, pp. 30793084.

[34] G. L. Plett, "Extended Kalman filtering for battery management systems of LiPB-based HEV battery packs: Part 3. State and parameter estimation," Journal of Power Sources, vol. 134, pp. 277-292, 2004.

[35] X. Rui, H. Hongwen, S. Fengchun, and Z. Kai, "Evaluation on State of Charge Estimation of Batteries With Adaptive Extended Kalman Filter by Experiment Approach," IEEE Trans. on Vehicular Technology, vol. 62, pp. 108-117, 2013.

[36] F. Auger, M. Hilairet, J. M. Guerrero, E. Monmasson, T. OrlowskaKowalska, and S. Katsura, "Ind. Applications of the Kalman Filter: A Review," IEEE Trans. on Ind. Electron., vol. 60, pp. 5458-5471, 2013.

[37] Z. Fei, L. Guangjun, and F. Lijin, "Battery state estimation using Unscented Kalman Filter," in Robotics and Automation, 2009. ICRA '09. IEEE International Conference on, 2009, pp. 1863-1868.
[38] L. Yuang-Shung and C. Ming-Wang, "Intelligent control battery equalization for series connected lithium-ion battery strings," IEEE Trans. on Ind. Electron., vol. 52, pp. 1297-1307, 2005.

[39] D. Belov and M.-H. Yang, "Failure mechanism of Li-ion battery at overcharge conditions," Journal of Solid State Electrochemistry, vol. 12, pp. 885-894, 2008/08/01 2008.

[40] C. Mikolajczak, M. Kahn, K. White, and R. T. Long, "Lithium-Ion Batteries Hazard and Use Assessment," Fire Protection Research Foundation, 1 Batterymarch Park, Quincy, MA 02169-7471, ReportJuly 2011.

[41] Z. Fei, L. Guangjun, and F. Lijin, "A battery State of Charge estimation method with extended Kalman filter," in IEEE/ASME International Conference on Advanced Intelligent Mechatronics, 2008. AIM 2008. 2008, pp. 1008-1013.

[42] K. Ogata, Discrete-time control systems. Englewood Cliffs, N.J.: Prentice Hall, 1995.

[43] S. M. Ross, A first course in probability, 8th ed.: Pearson Prentice Hall, 2010.

[44] B. D. O. Anderson, "Optimal filtering," J. B. Moore, Ed., ed. Englewood Cliffs, N.J. :: Prentice-Hall, 1979.

[45] G. Welch and G. Bishop. (2006, 7/12/12). An Introduction to the Kalman Filter. [pdf file]. 2012, 16. Available: www.cs.unc.edu/ $\sim$ welch $/$ media/pdf/kalman intro.pdf

[46] J. R. Macdonald, "LEVM/ LEVMW," 8.12 ed, 2013.

[47] A. Systems. (2009, 9/9/12). High Power Li-Ion APR18650 [Data Sheet]. Www.cosmoenergy.com/APR18650M1A Datasheet 2009.pdf

[48] A. N. L, "AUTONOMIE," ed: Argonne National laboratory, 2010.

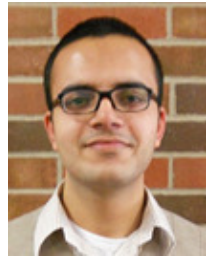

Amardeep Sidhu received his B.S. in Mechanical Engineering from Pune University, India, in 2007. He received his M.S. in Mechanical Engineering from Purdue University, Indianapolis, in 2013. From 2007 to 2011, he worked as Design \& Assembly Engineer with multinationals in Pune, India. He is currently working as HIL Test Engineer at Caterpillar Inc., Peoria, IL. His research interests include fault diagnosis, system modeling, adaptive control, and system identification.

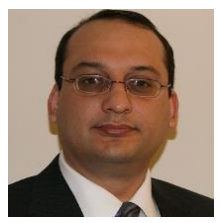

Afshin Izadian (SM'04-SM'10) received the M.S. degree in Electrical Engineering from Iran University of Science and Technology, Tehran, Iran, in 2002, and the Ph.D. degree in Electrical Engineering from the West Virginia University, Morgantown,WV, USA, in 2008. He was a Postdoctoral Researcher at the University of California, Los Angeles, CA, USA, in 2009. As an Assistant Professor, he joined the Purdue School of Engineering and Technology, IUPUI, Indianapolis, IN, USA, in 2009. He is the Founder and Director of Energy Systems and Power Electronics Laboratory (ESPEL) at IUPUI. His research interests are application of controls in energy systems, energy conversion, power electronics, and electric power systems. Dr. Izadian is a lifetime member of Etta Kappa Nu (HKN) and Tau Beta Pai (TBP).

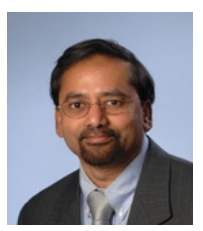

Sohel Anwar is Associate Professor in Mechanical Engineering Department at Purdue School of Engineering and Technology at IUPUI. He is also the chair of the graduate education and research committee and the director of Mechatronics Research Laboratory. He received MSME from Florida State University in 1990 and Ph.D. in Mechanical Engineering from University of Arizona in 1994. His R \& D work experience includes four years at Caterpillar, Inc. and five years at Ford Motor Company / Visteon Corporation. His research interests include wind turbine modeling and control, fault diagnosis of traction batteries, sensor development, and automotive control systems. Dr. Anwar is a Member of ASME. He is an Associate Editor of the IEEE Transactions on Vehicular Technology. 